\title{
Philosophiques
}

\section{Les autobiographies foetales masculines ou Jonas dans le ventre de la baleine}

\author{
Chantal Théry, Steven Morin, Sylvie Massé et Hélène Turcotte
}

Volume 21, numéro 2, automne 1994

Les femmes et la société nouvelle

URI : https://id.erudit.org/iderudit/027290ar

DOI : https://doi.org/10.7202/027290ar

Aller au sommaire du numéro

\section{Éditeur(s)}

Société de philosophie du Québec

ISSN

0316-2923 (imprimé)

1492-1391 (numérique)

Découvrir la revue

Citer ce document

Théry, C., Morin, S., Massé, S. \& Turcotte, H. (1994). Les autobiographies foetales masculines ou Jonas dans le ventre de la baleine. Philosophiques, 21(2),

503-523. https://doi.org/10.7202/027290ar
Résumé de l'article

Les quatre textes qui suivent tentent d'analyser dans la littérature québécoise et française récente (romans et essais, 1979-1989) les manifestations d'une société en mutation, désireuse ou non de rompre avec les stéréotypes de sexes, de revisiter et réconcilier féminin et masculin. Les écrivaines, avec quelques belles longueurs d'avance, continuent de vouloir à la fois le corps et l'esprit, la vie et la fiction, de jongler avec l'altérité et les identités plurielles et de travailler des textes ûctionnels, théoriques et incamés, qui prennent en compte le réel Leur travail de réflexion et de création ne cesse, en fait, d'interroger les artifices de l'art, les mensonges de la petite et de la grande histoire, et de prouver qu'on ne peut impunément dissocier éthique et esthétique! Le thème de l'autobiographie foetale qui hante la littérature masculine récente est très représentatif de la masculinité en crise; il cristallise ses angoisses, ses résistances ou sa nouvelle quête émotive, affective et corporelle.
Tous droits réservés (C) Société de philosophie du Québec, 1994
Ce document est protégé par la loi sur le droit d'auteur. L'utilisation des services d’Érudit (y compris la reproduction) est assujettie à sa politique d'utilisation que vous pouvez consulter en ligne.

https://apropos.erudit.org/fr/usagers/politique-dutilisation/ 


\title{
Les autobiographies foetales masculines ou Jonas dans le ventre de la baleine
}

\author{
par \\ Chantal Théry, Steven Morin, \\ Sylvie Massé et Hélène Turcotte
}

RESUME : Les quatre textes qui suivent tentent d'analyser dans la littérature québécoise et française récente (romans et essais, 19791989) les manifestations d'une société en mutation, désireuse ou non de rompre avec les stéréotypes de sexes, de revisiter et réconcilier féminin et masculin. Les écrivaines, avec quelques belles longueurs d'avance, continuent de vouloir à la fois le corps et l'esprit, la vie et la fiction, de jongler avec l'altérité et les identités plurielles et de travailler des textes fictionnels, théoriques et incarnés, qui prennent en compte le réel. Leur travail de réflexion et de création ne cesse, en fait, d'interroger les artifices de l'art, les mensonges de la petite et de la grande histoire, et de prouver qu'on ne peut impunément dissocier éthique et esthétique! Le theme de l'autobiographie fotale - qui hante la littérature masculine récente - est très représentatif de la masculinité en crise; il cristallise ses angoisses, ses résistances ou sa nouvelle quête émotive, affective et corporelle.

ABSTRACT : The following four-part paper try to analyse in recent quebecer and french litterature (novels and essays 1979-1989) the manifestations of a changing society, wether it be willing or not to break with gender stereotypes and reconcile the masculine/feminine dichotomy. Female writers still show leadership in wanting both body and mind, life and fiction; in thinking of alterity and multiple identities; and in working at fictionnal, theoretical and incarnate texts wich take reality into considerations. Their reflexion and creation in effect never stops questionning the artistic device, the lies of both small and big history to prove that we cannot inconsiderately dissociate Ethics from Esthetics! The fotal autobiography team now haunting recent male litterature is very representative of the masculinity crisis; it cristallizes his anguish resistances or his new emotive, affective and corporeal quest. 


\section{Introduction par Chantal Théry}

Dans le cadre d'un projet de recherche subventionné par le CRSHC ${ }^{\mathrm{I}}$, nous avons tenté de repérer à travers la littérature québécoise récente des personnages, des thèmes, des figures et des expressions inédites, novatrices, qui seraient autant d'alternatives pour sortir des stéréotypes de sexes.

Entre bien des sujets abordés - dont vous trouverez l'écho dans les articles de Steven Morin, Sylvie Massé et Hélène Turcotte ${ }^{2}$ - nous nous sommes interrogé/e/s sur plusieurs autobiographies fœtales masculines ${ }^{3}$ récemment apparues, dans le roman tant québécois que français : le thème n'est pas vraiment nouveau - que l'on se souvienne du vieux monologue, « Le fœtus », d'Yvon Deschamps - mais il connaît, par le nombre et l'ampleur des récits qui lui sont consacrés, une résurgence significative. Nous ne mentionnerons rapidement ici que la Couvade de Robert Baillie (Montréal, Quinze, 1980), Une belle journée d'avance de Robert Lalonde (Paris, Seuil, I986), La vie d'un bébé de François Weyergans (Paris, Gallimard, 1986) et La vie à rebours d'André Vanasse (Montréal, Québec/Amérique, 1987).

Dans quelle mesure ces autobiographies fœtales masculines ${ }^{4}$ représentent-elles une tentative de compréhension véritable du monde maternel ? une manifestation empathique et novatrice à porter au crédit des nouveaux pères? une volonté de ressentir de l'intérieur, de dire ses émotions, de lutter contre les stéréotypes socioculturels de sexes?

L'analyse de ces autobiographies fœtales masculines nous permet de les considérer comme des interrogations, des explorations, des réponses, dans le

I. Conseil de Recherches en Sciences Humaines du Canada, « Littérature québécoise contemporaine et histoire des idées : des voix originales pour sortir des stéréotypes de sexes », sous la direction de Chantal Théry (département des littératures, Université Laval), en collaboration avec Sylvie Massé, Bernard Ouellet, Hélène Turcotte et Steven Morin.

2. Déjà publiés, dans Féminisme et androgynie : explorations pluridisciplinaires, Les Cahiers du Grad n 4, Faculté de philosophie, Université Laval, 1990 : Chantal Théry, « De l'angélisme culturel à la culture musclée » (p. 55־57); Sylvie Massé, « Agir clandestin, définition hétérogène et sanction sociale : la perspective ludique des transgressions reliées au sexe dans le roman de Monique Proulx, Le sexe des étoiles » (p. 69-82); Bernard Ouellet, « Les stations de Dominique Larue : l'écrivain (mâle) québécois en procès » (p. 83-105). Chantal Théry, « Sujet humain non identifié : imaginaire en souffrance », dans L'Etranger dans tous ses états, Enjeux culturels et littéraires, sous la direction de Simon Harel, Montréal, XYZ, 1992, p. 6I-71.

3. Nous entendrons par « autobiographie fœtale » masculine, le livre d'un auteur masculin qui fait raconter par un narrateur-personnage omniscient le récit de sa vie intra-utérine.

4. La dernière en date est celle de Pascal Bruckner, Le Divin enfant, Paris, Editions du Seuil, 1992. 
sillage des recherches sur la vie du fœtus (photos intra-utérines/échographies), le « rebirth », les NTR lle mirage d'un bébé-éprouvette débarrassé de la mère/de l'homme enceint]; et, sur un mode plus dysphorique, comme indices de l'angoisse de la perte de pouvoir : inquiétude sur le donneur anonyme/la paternité/la patrilinéarité liée à l'insémination artificielle; dans le sillage aussi des gains du féminisme, de la mère monoparentale, du matronyme, du divorce et de la garde des enfants par la mère, de l'avortement (inoubliable affaire Chantal Daigle et Jean-Guy Tremblay) et, plus globalement, des menaces qui pèsent sur la vie et la « santé » de la planète.

Une belle journée d'avance du québécois Robert Lalonde est indéniablement le roman le plus novateur, même si la figure bien identifiée de la mère du narrateur se superpose trop à celle de l'amante innommée, si l'histoire dédoublée nous incite à mal départager le passé du présent, le narrateur-fœetus de son fœetus-enfant à venir et si, l'enfant conçu, le narrateur se perçoit plus facilement comme géniteur que comme père. Il n'empêche que le monde de Lalonde est holistique, que les descriptions de son fœtus de sexe indifférent ou double, sont merveilleuses de poésie, de sensibilité et de sensualité comme en témoignent, choisies entre bien d'autres, les citations suivantes :

Je serai peut-ètre homme et femme ? Je suis déjà mâle et femelle et plus encore, aquatique et filant vite, indéfini, ambigu, à cause du temps qui n'existe pas encore. Choisir est impossible. Je coule, je me faufile dans la tendresse brûlante de l'eau de ma mère. Je suis tour à tour dérobé et enrobé, petit mâle, petite femelle. Jaurai bientôt des branchies et un serpent d'os durs, je serai un petit poisson immobile et vertigineux. Mais j'aurai aussi du vent dans mon sac, une peau de méduse, et le sang de ma mère, longtemps opaque, me nourrira, sera mon premier lac rose, couleur du ciel de l'aube d'automne, et goûtera la mer inconnue. Je serai amphibie, ambivalent, ambigu. (p.153)

Même, un de ces jours où je serai stupéfié et malheureux d'être au monde et d'être deux, de ne pas trouver de place ni pour l'une ni pour l'autre des deux innocences en moi, j'irai au marais et j'aurai envie de l'engloutissement définitif, doux et frais. Un goût de boue et de néant, ce centre de la terre qui ne sera jamais l'enfer, pour moi, mais bien un repos mérité de racines, d'argile et de pierres précieuses. (p.170)

Dans la Couvade de Robert Baillie, le narrateur, professeur de son métier, futur père angoissé, transpose son angoisse dans l'écriture : la mère, coupable, banalisée, n’a presque pas la parole; malgré une certaine bonne volonté, Baillie ne sort guère des rails de la maternité symbolique : l'enfant demeure un prétexte au texte:

Je suis ce texte androgyne fécond. Je suis celui qui se fait lui-même, pêlemêle, des vies mêmes de ceux qui me refusent la vie. [...] Mon père et ma mère m'ont laissé pour héritage leurs vies mensongères. Ils ne sont pas morts, mais ils n'existent plus. J'ai dû coudre patiemment l'édifice de neuf mois d'attente. Une maçonnerie de dentelle. Cela m'a pris un siècle, toute ma substance. Ma mère et mon père, ces personnages romanesques, ils m'ont laissé une œuvre disparate, mais faite pour être rassemblée. Si j'étais le poète, je dirais : rapaillée. Mais je ne suis pas poète ni chanteur de charme. 
Je suis l'humble lecteur d'un texte multiple à réinventer, à reconstruire. C'est d'une architexture qu'il s'agit. (p. 259)

Nous avons tout le temps devant nous et nous sommes seuls avec ce qui nous hante. Ce siècle ou ce livre que nous entrevoyons, je le prends, je le garde. C'est à nous maintenant de parler. Ce sera mon chant, ma plainte, ma reconnaissance. Mon prétentieux, mon éternel moi. MON LIVRE. Notre livre. Car tout est à faire. Les enfants. Les romans. L'amour. Les enfants d'amour, les romans d'amour. (p. 260)

Dans la Vie à rebours d'André Vanasse, la compagne du personnage de l'écrivain se retrouve assez magiquement enceinte de plusieurs mois du double de l'écrivain. Il s'agit en fait d'une grossesse nerveuse qui se termine pourtant par une sanglante fausse-couche. La jeune femme désespérée se pendra, une poupée entre les bras... Dans beaucoup de nos romans d'auteurs masculins, la grossesse se solde souvent par un avortement, une fausse-couche ou une grossesse nerveuse qui en disent parfois beaucoup moins sur le refus de l'enfant que sur celui de la paternité. L'écrivain d'une Vie à rebours se suicide dans le Grand Nord en se noyant au soleil couchant, symbole de la fusion maternelle, d'un retour à la vie intra-utérine, redevenant ainsi l'enfant de sa mère, son propre enfant.

Dans la Vie d'un bébé, le français François Weyergans nous révèle explicitement, atténuées par ses pitreries de clown triste, ses angoisses. Il associe le big bang de l'origine ou de la fin de la Terre à la vie des fœetus : « Du photon au fœtus... [... J'ai escamoté le big bang. Eh oui, le gazouillis d'un petit fœtus est venu remplacer le silence éternel des espaces infinis. » (p. 156). L'avortement et les NTR l'obsèdent : l'éditeur souligne que le roman a été «salué à la fois par la presse littéraire et par des médecins et des gynécologues » adeptes sans doute du mouvement Pro-vie; le narrateur-fœtusautobiographe précise que « Sa mère aurait pu, tout à coup, vouloir se passer de lui. Ou lui d'elle. » (p. 68-9), que « Les fœtus sont à la merci de n'importe quoi. » et que, nouvelle Odyssée, « chaque fœtus est Ulysse, l'homme aux mille tours » (p. I43), qu'« un fœtus ne meurt que si c'est lui qui le décide », que « les fœetus mâles disparaissent plus facilement que les femelles » (p. 200), que « les ventres des femmes enceintes devraient devenir transparents. », que « L'avenir de la planète est dans les mains des fœetus » et que le XXI ${ }^{\text {e }}$ siècle ne sera pas ou verra la création d'un « ordre maçonnique des fœetus » en faveur d'« une humanité fraternellement organisée. » (c'est moi qui souligne, p. 208)! Je vous ferai grâce ici des stéréotypes machistes du texte ${ }^{5}$ !

5. La fécondation est racontée du point de vue des spermatozoïdes sur le vieux fond musical du « bravissimo spermatissimo » de Ricet Barrier; le fœtus est un intellectuel, un garçon nommé Jules, soucieux de l'avenir de son pénis et de ses testicules; sa mère aime des chansons un peu bêtes, « avant que j'arrive, elle avait sûrement moins d'idées », etc. Le narrateur parodie : «J'attends le Messie de Prudence Péronnelle. », et le fœetus joue à l'enfant divin ou au clown en habits de lumière qui surgit le jour de sa naissance comme d'un coffre-fort ou d'une boîtesurprise... Ce qui peut sauver Jules est inscrit comme un rébus dans son nom de famille : Aurel-Ladouceur/aurait la douceur... 
Les autobiographies fœtales masculines, indices d'une Crise de la masculinité, gagnent à être analysées d'un point de vue psychanalytique. Steven Morin perçoit heureusement dans cette forme de régression le signe d'une R/évolution.

\section{Retour à la mère : la régression vers l'évolution par Steven Morin}

Elisabeth Badinter, dans son essai $X Y$ de l'identité masculine, affirme que

[...] le mystère masculin demeure : le continent noir, cont-airement à ce que disait Freud, ce n'est pas la femme. C'est l'homme. Il y a encore peu de temps, la masculinité paraissait aller de soi : lumineuse, naturelle et contraire à la féminité. Nul ne songeait à questionner l'homme ! Les trois dernières décennies ont fait voler en éclats des évidences millénaires. Parce que les femmes ont entrepris de se redéfinir, elles ont contraint les hommes à en faire autant. (p.io)

C'est dans cette entreprise de (re)définition que plusieurs auteurs québécois ont eu recours à ce que nous avons appelé l'autobiographie fœale. Alors que l'homme traditionnel se sépare de la mère et de toute attitude à connotation féminine afin de se définir comme mâle, plusieurs auteurs d'ici ont préféré la symbiose avec la mère afin de retracer leur histoire et leur origine. Pourtant, la psychanalyste Lydia Flem, dans son article : « Le stade du cow-boy », publié dans la revue Le genre humain ( $\mathrm{n}$ ' Io) consacré à la masculinité, le confirme:

Né dans le giron de sa mère, nourri à son sein, élevé dans ses larges jupes, le garçon se trouve nécessairement imprégné par cette première symbiose avec la sphère féminine maternelle. Alors, pour acquérir la marque de son propre sexe, il lui faut s'en dégager, s'en dissocier, s'en arracher même. L'identité sexuelle de l'homme n'est pas en prolongement de celle de sa mère, mais bien en rupture. Fragile, l'identité masculine est à conquérir. Si le féminin est, d'emblée, le masculin, lui, se doit de faire pour être (p. Io6 ).

C'est ainsi que les psychanalystes contemporains ont défini le comportement masculin tel que nous pouvons l'observer dans toutes les cultures. Cependant, ce processus peut s'avérer dangereux. S'il est essentiel pour l'homme de se dégager de l'emprise maternelle afin d'acquérir son identité, il ne doit pas néanmoins s'amputer des valeurs dites féminines ou maternelles, telles que l'émotion, la sensibilité, la compassion, l'échange affectif.

Le retour à la mère manifeste, chez nos auteurs, le désir de repossession de ces valeurs humaines, encore trop stéréotypiques, associées au féminin et au monde maternel. Encore ici, c'est par la mère et les femmes que nos écrivains tentent de combler leurs manques. Ils projettent sur elles les émotions qu'ils ne peuvent introjecter, faire leurs ou exprimer avec leur corps, qu'ils ne peuvent partager à deux, comme la femme qui porte son enfant. C'est donc sur la symbiose mère-enfant que sera projeté l'idéal masculin nouveau; le paradis perdu loin des guerres et de la rationalité du monde patriarcal. Bref, nos écrivains se cachent dans le « ventre à maman » à la fois pour dénier leur réalité, 
incapables de porter les habits trop serrés de l'homme traditionnel, et pour y puiser une force régénératrice, comparable au phénomène du « rebirth » ou de l'analyse psychanalytique où l'homme, à travers l'introspection, peut maintenir le lien avec l'enfance et l'inconscient afin de renaître avec plus de vigueur.

De plus, nous avons remarque que ces tendances psychotiques (retour fantasmatique à la mère) apparaissaient à la suite d'un ou de plusieurs événement(s) déclencheur(s) qui perturbai(en)t la vie du héros comme la grossesse de la compagne du narrateur dans Une belle journée d'avance de Robert Lalonde. Dans Une vie à rebours d'André Vanasse, le héros se réfug:e symboliquement dans le ventre de sa voisine, après son implication dans une grève illégale. L'homme-têtard de Maurice Champagne, quant à lui, régresse à la suite de l'avortement de sa compagne. A cause de ces événements perturbateurs et de la faiblesse du moi et des mécanismes de défense, nos héros vont se réfugier dans le monde maternel. C'est ainsi qu'apparaît le phénomène de régression.

Le ventre féminin est sans aucun doute le lieu de prédilection de nos auteurs, exprimant ainsi, entre autres, la fameuse envie de l'utérus chez l'homme, telle que l'explique Christiane Olivier dans son célèbre ouvrage Les enfants de Jocaste. En effet, les hommes n'ont-ils pas toujours tenté de contrôler le pouvoir fascinant de la procréation? Symptôme d'une envie, d'une jalousie, ce contrôle exercé sur la maternité révèle leur crainte de n'être pas reconnus dans leur rôle de géniteurs. Quoi qu'il en soit, c'est sur le ventre maternel que sont projetés les aspirations les plus pures, les désirs les plus ardents et les réalisations les plus audacieuses. Si le corps des femmes fut de tout temps glorifié, c'est qu'il est du côté de la vie. Les hommes, quant à eux, conçoivent leur corps comme un lieu stérile, du côté de la mort, dans lequel l'émotion peut difficilement s'éveiller.

Rien de plus normal queles femmes se préoccupent de leurcorps, dit Ariane Émond de La vie en rose. Le corps féminin est médicalisé : il y a dès l'âge de I2 ou I3 ans les menstruations, puis la contraception, les accouchements, la ménopause, le lifting, etc. Les femmes sont proches de leur corps et attentives à leurs fonctions biologiques.

Georges-Hébert Germain, dans son article « Le syndrôme du bourdon », publié dans l'Actualité d'avril Ig84, poursuit :

L'homme par contre est resté (ou devenu) d'une pudeur maladive. Il ne sait pas parler ouvertement et simplement de son corps, sauf d'un point de vue strictement scientifique ou par le biais de ses fantasmes. On imagine difficilement une publication « masculiniste » dans laquelle les hommes parleraient de leurs érections, de leurs scrotums, de leurs impuissances et de leurs orgasmes, etc. (p. 46).

Dans La certitude dêtre mâle?, un essai québécois sur la condition masculine publié en 1980, Réginald Richard, dans son article intitulé « L’homme en mal de corps », écrit:

L'homme a toujours trouvé assez étrangères à sa personnalité, cette proximité de la femme de son corps et cette hantise de parler de ses 
émotions. Il s'y accommode, mais ne vit pas dans ses tripes l'ivresse de dire ses sentiments, cette urgence d'exprimer son vécu. C'est toujours avec un peu de honte et de pudeur qu'il tente d'en parler, toujours maladroitement, comme si on lui posait une question qui n'est pas la sienne, comme si on interrogeait un vécu qui est étranger. Si l'homme est moins proche de ses sensations corporelles que ne peut l'être la femme, c'est que contrairement à celle-ci, son corps est opaque et sans espace intérieur. Le corps de l'homme est silencieux; pas de cycle menstruel pour faire émerger la sensation de transformation, pas de grossesse, pour éprouver un espace intérieur où peut se loger un autre soi, pas de cavité vaginale où la sensation d'une entrée dans un dedans est possible, pas de cavité utérine où l'expérience du vide et du plein peut être faite. Le corps de l'homme est un corps qui ne se vit pas comme dedans, espace intérieur où se logerait une vie émotionnelle, des sensations très précises. Assez peu de transformation biologique dérange son fonctionnement habituel et régulier au point d'y faire naître une vie émotionnelle. Le corps de l'homme est un corps fermé sur luimême, imperméable à l'intrusion de l'autre, parce que en lui aucun espace intérieur n'est susceptible de recevoir quelque chose, sauf dans l'expérience de la nutrition (p.78-79).

Maurice Champagne, quant à lui, définit, dans son essai-fiction L'hommetêtard, le ventre de l'homme comme « un petit conduit de respiration affective ». Dans sa théorie de la masculinité, le psychanalyste jungien Guy Corneau met l'accent sur la dichotomie corps/esprit chez les hommes. En effet, si l'homme pense beaucoup avec la tête, il pense difficilement par le corps. Le corps devient donc une structure autonome, entre la tête et le sexe, où le ventre est un endroit clos où s'infiltrent la plupart des maladies psychosomatiques masculines reliées au pancréas, aux reins, au cœur, à la prostate, etc. Le corps devient le lieu de la mère et la tête le lieu du père, et entre les deux figures, donc également entre les deux espaces, apparaît une scission. L' « absence » de corps chez les hommes signifie que leur corps appartient encore à la mère. A travers la surmusculation et la compétition des corps, l'homme tente de se détacher du corps de la mère afin de s'affirmer dans/par un corps autonome et «mâlifié». Les sports masculins et les fondements de l'idéologie patriarcale sont une tentative d'autocastration violente, une protestation virile contre la mère. Une forme d'initiation active et pratiquée en négation contre les valeurs des femmes certes, mais d'abord contre celles de la mère. En effet, l'individuation, l'autonomisation et le recours à des formes d'initiation souvent dénaturées sont le propre de l'homme en quête urgente d'une identité. Voilà où l'absence de père a conduit les fils en manque de modes d'identification masculine positifs.

C'est pourquoi le ventre de la femme est idéalisé, il est le centre de projection de leur propre émotivité, de l'aspect de la vie et de la création. Un ventre survalorisé par rapport à une dévalorisation masculine, source d'une insécurité face à leurs propres émotions. L'imaginaire masculin est davantage du côté de la projection vers l'extérieur, celui du corps social par opposition ici au corps propre. Cette peur de l'onirisme et de l'intégration du moi (des émotions) est due principalement à une absence de figure paternelle initiatrice, 
d'une part, au langage de leur spécificité corporelle, et d'autre part, au langage affectif.

En effet, la quête de l'initiation est un thème récurrent chez nos auteurs. Celle-ci s'exerce à travers les transgressions spatio-temporelles, l'adultère, la fuite, l'homosexualité, la rébellion, etc. Une identité fragmentée tente de se rassembler en un TOUT au travers des rites initiatiques souvent étranges mais toujours orientés vers la transgression et la quête. Par exemple, la quête du frère comme substitut paternel dans Volkswagen Blues de Jacques Poulin. Dans Une belle joumée d'avance de Robert Lalonde, le personnage de Gilles, véritable figure chamanique, initie le narrateur au langage de la nature et des émotions. C'est également cette figure paternelle positive qui expliquera au narrateur la fonction régénératrice de la régression vers l'univers fœtal :

En bas, c'est le centre de la terre. On descend, on passe un jour complet dans la terre et quand on remonte, on est lavé de l'âge, du temps, du pourrissement (p. 105).

La régression dans l'univers fœtal est donc conçue telle une forme d'initiation purificatrice, une quête d'identité à travers la renaissance, lieu où la figure paternelle et le monde maternel se rejoignent en une complémentarité, en une non-dissociation entre le vécu féminin et le vécu masculin. La figure de l'absolu androgyne est ici la voie du salut de l'homme, la réconciliation entre le corps et l'esprit, entre la pensée et l'émotion. Gilles invitera le narrateur à l'initiation ultime : se tenant nu sous la pluie, les bras levés entre le ciel et la terre, le sujet est initié à l'Ordre de la nature, il découvre les sensations du corps et la pureté de l'enfance. Enfant du ciel et de la terre, enfant de la nature, l'homme se découvre ainsi nu, lavé des identifications secondaires, des stéréotypes patriarcaux. Il tente de se redéfinir dans l'essence même de la nature, il est Humain avant tout.

Comme Badinter le souligne, toujours dans son essai $X Y$ de l'identité masculine :

[...] la relation première à la mère est la condition même de l'identité humaine du mâle. Si cette relation n'est pas bonne ou si l'identification n'est pas possible, l'enfant aura toutes les difficultés à devenir un mâle humain (p. 80). [...] la masculinité n'est pas une essence, mais une idéologie qui tend à justifier la domination masculine ( p. 48 ).

Il n'y a pas de modèles mâles universels ou intemporels. En cette crise de masculinité, où les hommes doivent se définir dans la solitude, par le questionnement et l'introspection, toutes les possibilités sont admises et nombre d'auteurs québécois empruntent les voies de l'identité plurielle et de la transgression. L'écriture devient ainsi l'initiation par laquelle ils se redéfinissent à l'infini, en quête d'un lieu commun où l'identité se fonde en un TOUT. Le ventre maternel ne sera qu'un espace parmi d'autres habité par l'imagination masculine afin de résoudre son mâle-d'être... Cependant, cette tentative est essentiellement positive.

Comme le remarque Elisabeth Badinter : 
Pour se laisser aller à ces fantasmes régressifs, il faut déjà avoir pris quelques distances avec ses angoisses. Peut-être aussi l'actuelle remise en question de la masculinité et de la féminité relâche-t-elle le nœud de la répression qui, il y a encore vingt ans, étranglait l'homme. Mais tous ne sont pas capables de cette lucidité sur eux-mêmes. Les plus fragiles, les plus douloureux aussi, ne peuvent maintenir leur masculinité et lutter contre le désir nostalgique du ventre maternel que par la haine du sexe féminin (p. 9i).

Aussi, si la régression est, à certains points de vue, contraire à l'évolution, elle est néanmoins associée au repos du guerrier. Elle s'inscrit comme un temps de réflexion, une pause dans la course folle des hommes vers la Vérité. Guy Corneau écrit, dans son célèbre essai Père manquant, fils manqué :

La vie mutile sauvagement nos idéaux, comme si dans l'étreinte noire de l'existence matérielle un secret devait être découvert, un secret qui semble se gagner par la souffrance. La mutilation de l'accident, comme celle de la solitude ou de la rencontre avec soi représente une sortie du paradis terrestre, nous voici chassés du ventre de la mère-monde. Où es-tu mère de toutes les bontés, douceur infinie ? Où es-tu vie fœtale, tropicale et douce? Pourquoi faut-il vivre les armes à la main? (p. 154).

Toutefois, cette problématique du retour à la mère afin de se redéfinir et de créer une nouvelle figure paternelle positive, n'est pas strictement masculine. Si les hommes doivent couper symboliquement les liens avec la mère afin de devenir « mâle » à part entière, les femmes ont dû également faire de même pour se définir en tant que « femme nouvelle», adaptées à la société moderne. Les mères traditionnelles n'ont plus été pour elles des points de repères. De mères « dépassées » par rapport auxquelles elles ont dû se contre-identifier, les femmes n'ont guère eu de choix que de sacrifier certaines valeurs maternelles afin de s'imposer en tant qu'êtres pensants et créatrices à part entière, tout comme les hommes. De plus, Julia Kristeva affirme dans Soleil noir :

Pour l'homme et pour la femme, la perte de la mère est une nécessité biologique et psychique, le jalon premier de l'autonomisation. Le matricide est notre nécessité vitale, condition sine qua non de notre individuation. pourvu qu'il se passe de manière optimale et puisse être érotisé : soit que l'objet perdu soit retrouvé comme objet érotique (c'est le cas de l'hétérosexualité masculine, de l'homosexualité féminine), soit que l'objet perdu soit transposé par un effort symbolique incroyable et dont on ne saurait qu'admirer l'avènement, qui érotise l'« autre » (l'autre sexe, dans le cas de la femme hétérosexuelle) ou bien qui métamorphose en objet érotique «sublimé » les constructions culturelles (on pense aux investissements, par les hommes et par les femmes, des liens sociaux, des productions intellectuelles et esthétiques, etc.) ( p. 38).

Toutefois, la mère doit être ÉGALEMENT récupérée dans ses valeurs positives et humaines inhérentes et non seulement en tant qu'objet érotique projeté ou sublimé. Les féministes ont dû se battre sur le même terrain langagier que celui des hommes afin de se faire reconnaître et ainsi, prendre place dans une société encore, et malheureusement, dictée par un patriarcat désuet. La parole des femmes modernes emprunte donc un discours le plus souvent théorique, intellectuel ou politique, le seul langage encore valorisé par les principales institutions. Combien de femmes ont dû se couper de leur 
sensibilité, de leurs émotions, afin d'adopter une attitude intellectuelle avec laquelle elles revendiquent le droit à l'égalité et au respect de leur différence? Voilà peut-être pourquoi quelques théoriciennes féministes, souffrant également d'une coupure narcissique, se sont senties interpellées par la crise de masculinité (Rosalind Miles, Elisabeth Badinter, Denise Bombardier, etc.). A quel point n'est-ce pas là un miroir de leur propre blessure ? Comment alors expliquer cet intérêt soudain, ce regard de féministe bien-aimante sur l'autre sexe? Les femmes d'aujourd'hui n'ont-elles pas aussi à repenser leur identité, et ce, une fois de plus, exaspérées par l'invulnérabilité émotive de la superwoman ? N’ont-elles pas à retourner également en arrière afin de récupérer des valeurs humaines sacrifiées trop vite à la modernité?

Cette thématique du retour à la mère et le remodelage d'un nouveau père, est également notre « petite histoire » des vingt dernières années. En effet, après la révolution féministe et la diffusion de l'utopie matriarcale, voici, quelques années plus tard, l'apparition d'un thème novateur : l'espoir en un renouveau paternel. Comme le Québec fut l'un des lieux les plus sensibilisés aux discours féministes, il n'est pas surprenant que ce soit précisément chez nous que l'appel aux nouveaux pères ait été le plus percutant et le plus sensiblement exprimé, et ce, par des hommes pro-féministes (Guy Corneau, Marcel Champagne, Marc Chabot, etc.). En effet, ce sont les remises en question apportées par le féminisme qui ont obligé les hommes à se (re)créer une condition appelée à évoluer et à se sortir des stéréotypes socio-culturels. Comme quoi les mères et les femmes, héritières des origines, appellent à la naissance et à la renaissance. C'est par elles que se construit un monde nouveau qui ne peut toutefois pleinement évoluer vers un équilibre sans l'apport de l'Autre différencié, le signifié du Père. La corrélation de l'un et de l'autre, la fusion d'un matriarcat et d'un patriarcat, tous deux redéfinis, non en opposition l'un avec l'autre à l'intérieur d'un discours s'appuyant sur le clivage des valeurs, mais bien en fonction de cette certitude de l'aliénation de l'un sans l'autre, nous permettra sans doute d'en finir enfin avec cette interminable guerre idéologique des sexes. C'est sûrement dans/par l'espace maternel, par ce point d'origine des deux sexes, à l'intérieur de ce lieu commun qu'ils tenteront sans cesse de recréer fantasmatiquement, que se rejoindront enfin « féminité » et « masculinité » en une totalité symbolique.

La psychanalyse nous a montré l'importance fondamentale des rôles maternel et paternel, la nécessité de leur présence affective dans la structuration de l'identité. Il en est de même pour leur projection en macrocosme que sont le matriarcat et le patriarcat, dont le parallélisme et la fusion sont fondamentaux pour le mieux-être de la société. Ces deux rôles/pôles doivent être identifiés dans leur importance égale, tout en soulignant l'urgence de leur réconciliation. Ne porte-t-on pas en soi les marques d'une mère et d'un père? Les conflits psychiques et sociaux ne sont-ils pas le fruit d'une guerre des sexes, d'un père et/ou d'une mère négatif/tive avec qui la réconciliation est fondamentale pour notre mieux-être, pour le développement de toutes nos 
potentialités, afin de libérer toutes les valeurs enfouies sous ces figures bafouées, refoulées, rejetées?

Le retour à la mère doit être dorénavant défini comme une régénération nécessaire et inhérente à l'évolution personnelle et sociale. Ce qui, à première vue, semblait d'emblée être une problématique strictement masculine reliée à une crise d'identité, devient d'un intérêt universel, concernant les deux sexes. Il faut (re)découvrir les qualités maternelles afin de se réconcilier avec le père absent ou mis à mort, le signifiant de l'Autre, du Social. C'est en puisant dans la Mère positive des forces affectives que nous pourrons renouer avec le Père/ le Social, où, avec la figure stricte du paternel-Loi, s'harmoniseront un langage d'amour et un sourire humain. Loi de Société et Loi de la Nature, langage intellectuel et langage sensible, ambition et affectivité, père et mère, homme et femme, ne seront plus des qualificatifs ou des entités clivés et discriminés. Ils seront considérés comme inhérents à la nature humaine. Nous avons donc besoin de discours nouveaux, parlés par des hommes et des femmes, complices, réconciliés dans l'adage « l'Un et/est (naît) l'Autre » et avec, en soi, la (re)valorisation des figures parentales positives, qui, dénonçant le clivage des valeurs, annuleront ces termes piégés que sont « masculin » et « féminin », pour avancer vers une société nouvelle et pacifiante. Cessons également d'utiliser le terme utopie, ce mot sans espoir teinté d'impossibilité. Dans un esprit positif et constructiviste, décrivons les prémices d'une société à venir...

\section{Solutions}

L'ambivalence : toute son affaire est de savoir si les parents sont bons ou mauvais.

L'ambiguïté : toute son affaire est de savoir si les parents viennent d'ailleurs ou s'ils viennent de soi.

Ambivalence résolue : les parents sont à la fois bons et mauvais.

Ambiguilté aboutie : les parents sont venus d'ailleurs et viennent de soi.

(Paul-Claude Racamier, Le génie des origines, p. 39I)

\section{Réflexion sur le rapport des femmes à la création. Voies/voix exploratoires par Sylvie Massé et Hélène Turcotte}

Notre analyse, qui comporte plus d'hypothèses que de réponses, se veut une réflexion sur le rapport des femmes à la création et subsidiairement (ou parallèlement) sur leur rapport à la vie, et ceci à travers leurs représentations du monde. Certaines interrogations sont à l'origine de notre réflexion : les femmes participent-elles de la culture dominante, culture masculine par définition (les deux sexes (fort et faible) partageant ainsi la même idéologie) et la représentation que les femmes se font du monde est-elle la même que celle des hommes? En 1978, Françoise Héritier, anthropologue, affirmait au cours d'un entretien avec Evelyne Sullerot que croire en l'existence d'une culture 
féminine et d'une culture masculine est un a priori féministe tout à fait faux du point de vue anthropologique :

[...] la représentation que les femmes ont du monde est la même que celle que les hommes en ont ${ }^{6}$.

Pourtant, l'examen comparatif de textes d'auteurs masculins et de textes d'auteurs féminins favorise la conclusion contraire. Ainsi, comme le soulignait Jeanne Lapointe, la littérature, qui se targue généralement d'être d'avantgarde, novatrice, est tout à fait conservatrice en ce qui concerne la relation homme/femme, « les changements de perspectives, dans ce domaine, (étant) généralement le fait des femmes ${ }^{7}$. De même, l'analyse de textes ayant comme thème la procréation, telle l'autobiographie fœetale ${ }^{8}$, tend bien à prouver que la représentation que les hommes et les femmes se font du monde n'est pas la même.

Parlons donc d'une culture au féminin, culture de l'hétérogène, de « l'autre ». Mais une fois cette constatation faite, nombre de questions demeurent en suspens. Comment peut-on définir la culture au féminin, le concept de culture étant par ailleurs fort large? En quoi se distinguerait-elle d'une culture plus vaste? Les textes d'auteurs féminins, circulant généralement dans le même environnement que les textes d'auteurs masculins, se trouvent rejetés ou reconnus par l'institution littéraire ou artistique, et, en ce sens, ils naissent et meurent dans un contexte culturel commun. La culture au féminin devrait donc être qualifiée de culture minoritaire et non pas de culture parallèle puisqu'elle « ne s'est pas développée parallèlement à la culture patriarcale ou en dehors d'elle mais en rapport constant avec elle, de sorte qu'elle ne peut pas s'en arracher sans s'amputer d'elle-même $»^{9}$. De plus, de quelle manière cette culture se définirait-elle à travers l'histoire? Une histoire que les femmes n'ont pas faite, à travers laquelle elles ne sont pas sujets, situées du côté de l'anonymat, de l'anhistorique, de l'histoire dite sans qualité, sauf pour quelques femmes qui, comme le disait Françoise Collin, sont « victorieuses » et

6. Evelyne Sullerot et al., Le fait féminin, Paris, Fayard, p. 398.

7. Jeanne Lapointe, « Perspectives féministes en littérature », Un savoir à notre image? Critiques féministes des disciplines (sous la direction de Roberta Mura), Adage. Montréal, ig9i, p. 38.

8. L'apparition de l'autobiographie fœtale dans la littérature québécoise est davantage le fait des auteurs masculins, comme le soulignait Chantal Théry dans un article intitulé « De l'angélisme culturel à la culture musclée » : « Parallèlement aux manipulations génétiques de la mère idéale, c'est-à-dire le savant-démiurge. ou au projet futuriste de l'homme enceint, on observe d'ailleurs dans la littérature l'émergence d'autobiographies masculines particulières, axées sur ce quej'appelle « la vision fœtale », c'est-à-dire le récit de la vie intra-utérine par un narrateur omniscient [...] », dans Féminisme et androgynie : explorations pluridisciplinaires, Les Cahiers du Grad, $\mathrm{n}^{\circ}$ 4, 1990, p. 6o-6r.

9. Françoise Collin, « Il n'y a pas de cogito-femme », Lémergence d'une culture au féminin. sous la direction de Marisa Zavalloni, Ed. Saint-Martin, Montréal, 1987. 
« émergent « comme des hommes » sur la scène publique qui est toujours scène du pouvoir $|. .|.\rangle^{\text {IO }}$. La culture des femmes est ouverte sur l'immémorial, sur la trace et non sur la marque et les femmes font l'histoire « de ce qui échappe à l'histoire, et qui ne se nouerait pas dans le nom mais dans l'innommé $»^{11}$.

La création au féminin est justement le reflet de cet « innommé », originant d'une mémoire « autre » et recréant le monde « autrement ». Reprenant ici les idées de Luce Irigaray, nous pourrions dire que la création amène les femmes à « se définir un espace qui leur permette d'émerger comme sujet $\rangle^{\mathrm{I}^{2}}$. Mais elles n'ont pu accéder à la création artistique et littéraire qu'après un long cheminement, cheminement qui n'a pas été exempt de luttes. L'Histoire les a trop souvent laissées pour compte et il a fallu attendre la révolution féministe pour que soient interpellees les valeurs traditionnelles et mythiques. Nous nous sommes donc intéressées plus particulièrement à la littérature de la décennie 80 , le corpus faisant l'objet de notre analyse regroupant des œuvres de femmes publiées entre ig79 et I989. Cette période littéraire pourrait être qualifiée de post-féministe en raison de la transformation des thèmes revendicateurs des années 1970. Comme le soulignent Gaston Miron et Lise Gauvin dans leur ouvrage Ecrivains contemporains du Québec depuis 1950 : «Vers les années quatre-vingt, on passe à des œuvres plus feutrées, explorant les registres de l'intime $[. ..] \gg^{\mathrm{I}}$. Dans la période qui couvre les années quatrevingt, cette écriture de femme, écriture des métamorphoses, reflète le malaise d'une société en mutation. Et nous nous sommes tournées vers ces œuvres pour explorer, découvrir les voies empruntées par les écrivaines pour parler de création. Après les années de dénonciation de toutes sortes et de revendication du plaisir d'écrire, où en est rendue la création littéraire des femmes? Si l'écriture masculine fait surgir des thèmes nouveaux, partiellement innovateurs, qu'en est-il de l'écriture féminine?

Io. Françoise Collin, « Voyage à Lausanne ou La marque ou la trace », Penser au féminin, Les Cahiers internationaux du symbolisme, n 65-66-67, 1990, p. 51 .

II. Ibid., p. 54 .

12. Cette idée de Luce Irigaray est rapportée par Marie Denis dans un article intitulé « Corps et âme »: « [...] Luce Irigaray fut parmi les premières à donner aux femmes à la fois la connaissance et la fierté de leur être au féminin, à définir un espace qui leur permette d'émerger comme sujet, seule condition d'un face à face humain, d'une différence des sexes libre et réciproquement acceptée. Aujourd'hui, découragée peut-être de voir les humains si peu attentifs à des voix encore trop silencieuses, elle jette un cri d'alarme contre une « pensée au neutre », mortifère pour le genre humain. Le monde meurt de ne pas avoir connu ses genres, explique-telle, de les avoir niés, l'un s'affirmant sans l'avouer (le fameux masculinisme !) et empêchant ainsi l'autre de venir à naître comme parole. », dans Penser au féminin, op. cit., p. 43 et 44 .

13. Gaston Miron et Lise Gauvin, Ecrivains contemporains du Québec depuis I950, Paris, Seghers, 1989, p. 20. 
Les écrivaines des années quatre-vingt innovent de plus d'une façon. D’abord par la création de personnages nouveaux. Dans Le Sexe des Etoiles ${ }^{\mathrm{I}}$, Monique Proulx dépeint deux êtres transcendants : Camille, l'adolescente rebelle, enthousiasmée par l'astrophysique, porte un regard holiste sur le monde ${ }^{15}$ et Marie-Pierre, transsexuel et père-mère de l'adolescente, qui rejette les valeurs antinomiques de la sociéte $e^{16}$. Dans Le dernier recours ${ }^{17}$ de Christine L'Heureux, Jacques, un jeune médecin, désireux d'adapter la société à une réalité nouvelle, devient le premier homme enceint. Plusieurs personnages apparaissent hors-normes, peu soucieux de se soumettre aux règles, conscients d'être des marginaux au sein d'une société sans imagination, encore éminemment stéréotypée et rétrograde en ce qui concerne les rapports de sexe.

Les écrivaines innovent également par leurs thèmes. Parmi ceux-ci, implicite dans plusieurs œuvres, l'altérité est l'un des enjeux de l'écriture des années 8o. Ainsi, la confrontation de Jacques avec son double féminin dans $L e$ Dernier Recours entraîne de très beaux discours sur cette idée. Mais d'autres avenues sont aussi explorées, qu'il s'agisse des sensations et de la littérarité du corps comme dans Autographie I. Fictions de Madeleine Gagnon (VLB éditeur, I982); la Lettre aérienne (Éd. du Remue-ménage, 1985) et Domaine d'écriture (Éd. NBJ, 1985) de Nicole Brossard, ou encore de maternité symbolique et d'androgynie comme dans Le Silence de la cité d'Elisabeth Vonarburg (Denoël, coll. Présence du futur, I98I); Ange Amazone de Yolande Villemaire (Les Herbes roubes, I982); Danseuses-mamelouk de Josée Yvon (VLB éditeur, I982); L'Androgyne de France Vézina (L'hexagone/théâtre, I983) et Des cailloux blancs pour les forêts obscures de Jovette Marchessault (Leméac, 1987). Le thème de l'androgynie permet aussi la quête d'une fusion originelle, d'un retour à l'autre et au même, à la figure du double, de l'âme sœur, du frère/sœur, des jumeaux véritables ou symboliques. Encore que le concept d'androgynie conduise quelquefois à la réappropriation du féminin par le masculin ou à une intégration bisexuelle.

A travers l'écriture du corps, une quête de soi s'instaure dans Nous parlerons comme on écrit de France Théoret (Les Herbes rouges, 1982), Le Traversier

14. Montréal, Québed/Amérique, 1987.

15. « Ainsi, Camille rejette les références théologiques, l'hégémonie du genre, et crée, à l'aide de son imaginaire, un univers holiste, vivant, en continuelle métamorphose, dans lequel le sexe n'a plus qu'une importance toute relative : '1.... il fallait parler et demander la réponse aux mystères et savoir d'où cela venait et comment et pourquoi la mort la vie le commencement des étoiles - de quel, de quel sexe êtes-vous ?". ». Sylvie Massé « Agir clandestin, définition hétérogène et sanction sociale » dans Féminisme et Androgynie : explorations pluridisciplinaires, op. cit., p. 79.

I6. «L'enjeu de sa recherche ne consiste pas à gommer toutes traces de sa nature sexuée, mais à chercher comment être vrai-e, "authentique", au travers de cette nature sexuée inaltérable, malgré les normes et les conventions. », ibid., p. 79.

17. Montréal, Libre Expression, 1984. 
d'Esther Rochon (Éd. de la Pleine Lune, 1987) et une nouvelle mythologie se cree dans Le Pique-nique sur l'Acropole de Louky Bersianik (VLB éditeur, 1979), La Mère des herbes de Jovette Marchessault (Quinze, I980), Du côté hiéroglyphe de ce qu'on appelle le réel (Les Herbes rouges, 1982) et La Constellation du cygne (Éd. de la Pleine Lune, 1985) de Yolande Villemaire, et dans La Femme de Sath d'Andrée-A. Michaud (Québec/Amérique, 1987). Chez plusieurs auteures, on sent le besoin d'exorciser les puissances masculines, de dénoncer la surdité du mâle.

A la recherche de leur identité, les personnages, en proie à des identités plurielles, se dédoublent ou se multiplient, engendrant un morcellement de l'individu/e: Le Double suspect de Madeleine Monette (Quinze, 1980), L'Cil dela nuit d'Elisabeth Vonarburg (Le Préambule, 1980), Les Funambules de Dominique Blondeau (Libre Expression, 1980), La Passion selon Galatée (Seuil, 1987) et Les Aventures de Pomme Douly (Boréal Express, 1988) de Suzanne Jacob. D'autres textes présentent des écrivains aux prises avec leur double dans un mélange de fiction et de réel. Il y a parfois osmose entre les êtres, ce qui débouche sur le thème de la gémellité, des jumeaux symboliques : Les Faux Fuyants de Monique Larue (Québec/Amérique, 1982), Sans cœur et sans reproche de Monique Proulx (Québec/Amérique, I984), et Renaissance en Paganie d'Andrée Ferretti (L'Hexagone, 1987). D'autres fois, l'éclatement de personnalité ou l'intertextualité entraînent une polyphonie de voix féminines : Le Long des paupières brunes de Rolande Ross (Quinze, 1982), Visions d'Anna de Marie-Claire Blais (Éd. Stanké, 198I), et Le Désert mauve de Nicole Brossard (L'Hexagone, 1987).

La quête de soi fait retourner certains personnages aux origines, à la matrice dans Contes intemporels (Amours) d' Hélène Ouvrard (Éd. Marcel Broquet, 1985), ils trouvent l'autre, la mère, comme dans Les Mensonges d'Isabelle de Gabrielle Poulin (Québec/Amérique, 1983), La Danse de l'amante de Madeleine Ouellette-Michalska (Éd. de la Pleine Lune, 1987), et La Mémoire à deux faces d'Esther Croft (Boréal, 1988), alors qu'il s'agit à d'autres moments d'une quête du père : Le Sexe des Etoiles de Monique Proulx (Québec/Amérique, 1987), Les dimanches sont mortels de Francine D'Amour (Guérin, 1987$)^{18}$. Nous assistons à un retour aux sources marines, à une régression océanique : Le Dernier Recours de Christine L'Heureux (Libre Expression, 1984), et Hommes de Carole Massé (Les Herbes rouges, I988). La dispersion de la personnalité amène l'écriture fœtale ${ }^{19}$ dans Laura Laur de Suzanne Jacob (Seuil, 1983), Sans coeur et sans reproche de Monique Proulx (Québec/Amérique, 1984), les Demoiselles de Numidie de Marie

I8. D'autres textes traitent spécifiquement de la problématique fille/père, tels La Fille de son père de Linda Schierse Leonard (Le Jour, 1990) et À propos de l'amour de Christiane Teasdale (Boréal, ig9i).

19. Jusqu'à présent, il existe très peu d'autobiographies fœtales écrites par des femmes, ce thème étant davantage privilégié par les auteurs masculins. On note tout de même quelques textes s'intéressant à la «vision fœtale » dans la prose féminine du Québec (cf. les titres cités dans le présent texte). 
Josée Thériault (Boréal Express, 1984), et Nobody de Carole Massé (Les Herbes rouges, 1985). Dans son roman la Cohorte fictive, Monique Larue reprend la dichotomie pro-création/création : «Serait-il possible que ce ne soit pas possible ? $\gg^{20}$. Dans un livre qui discute de la maternité littéraire, d'un combat parallèle contre le papier blanc et contre la délivrance du fœetus, de la contradiction du langage contre les contractions du corps, de l'opposition du livre de peau contre l'enfant de chair, de la difficulté de mener de front les deux délivrances, Monique Larue, entre déchirement et sérénité, préfère osciller entre les deux valences.

L'association des idées de création et de procréation peut paraître dangereuse. Certains, encore convaincus que la nature des femmes (en particulier leur fonction maternelle) justifie leur situation dans la société, utilisent cette association afin d'expliquer l'absence des femmes dans l'histoire intellectuelle et artistique. Ainsi, dans un ouvrage récent ${ }^{2 \mathbf{I}}$, Réelles présences, George Steiner écrivait que « la mimésis est une réappropriation », une lutte entre un créateur mortel et son rival divin. Et il ajoute, confondant, nous semble-t-il, hétérogénéité et mise à l'écart :

Il se peut, encore que la chose ne soit pas nécessaire, que tout modèle de la création comme étant un antagonisme, une lutte avec et contre « l'autre créateur », contienne un préjugé de sexe. [...] La capacité biologique de procréer, d'engendrer la vie qui est le propre de la femme, n'est-elle pas de quelque façon, à un niveau absolument essentiel à l'être de la femme, tellement créatrice, tellement épanouissante, qu'en comparaison, la création de personnes fictives qui est la matière même du drame et des arts plastiques, en pâlisse? L'expérience que fait une femme de la naissance de son enfant - expérience qui, par essence, est inaccessible à la compréhension masculine - peut-elle être tellement voisine de l'énigme, de la sacralité, de l'existence de la vie elle-même (« pourquoi n'y a-t-il pas rien?»), qu'elle exclut, ou presque, toute impulsion de rivaliser avec un « Dieu jaloux » qui me semble à ce point cruciale dans l'acte esthétique ${ }^{22}$ ?

Tout cela nous ramène à l'idée que les hommes créent afin de pallier leur incapacité de procréer (ce qu'ils font entre autres dans les autobiographies fœetales) et que les femmes enfantent mais ne créent pas. « Aux hommes la création, aux femmes la procréation? Aux hommes les romans et aux femmes les enfants? Aux hommes l'esprit et aux femmes le corps ${ }^{23}$ ? » Cette réappropriation dont parle Steiner est certes partie constituante de l'acte créateur mais ne s'effectue-t-elle pas différemment chez les créatrices, la représentation qu'elles se font du monde étant différente ? Les différences n'engendrent l'inéquité sociale qu'en raison de jugements de valeurs. Evaluant le pouvoir procréateur des femmes, les hommes les stigmatisent ou les encensent, leur déniant par association tout autre pouvoir créateur. Pourtant, la possibilité de

20. Monique Larue, La Cohorte fictive, Montréal, L'Etincelle, 1979, p. 68.

21. L'édition originale de l'ouvrage a été publiée par Faber and Faber, Londres, 1989.

22. George Steiner, Réelles présences. Les arts du sens, Paris, Gallimard, 199I, p. 246-247.

23. Nancy Huston, Jounial de création, Paris, Seuil, 1990. 
procréer des femmes ne les rapproche-t-elle pas d'un acte créateur, non plus rivalité Inous pensons ici au titre d'une œuvre de Madeleine Gagnon, Toute écriture est amour. Autographie 2 (VLB éditeur, I979)l et artifice d'une re-création du monde, mais acte créateur se voulant redéfinition de réalités multiples, d'une fusion corps/esprit redécouverte?

Ce sont du moins les idées que défend Nancy Huston dans son dernier ouvrage, Joumal de création, dans lequel elle parle des mensonges de la création, des artifices de l'art, dont ne sont pas dupes les créatrices :

[...] Comment se fait-il que le créateur soit l'Homme par excellence, au point que Beauvoir puisse affirmer que celui-ci n'a pas encore pu s'incarner dans la femme?

C'est que, bien sûr, l'art est une des manifestations les plus spectaculaires de la rupture entre l'humain et l'animal. L'art dit non lou tout au plus oui, mais) à la nature. S'érige contre elle. Déclare son indépendance par rapport à elle. A travers l'art, l'homme s'affirme non pas créature mais créateur ${ }^{24}$.

En posant comme prémisse que le temps se trouve inscrit dans le corps des femmes par différents facteurs physiologiques, la vie s'originant à l'intérieur d'elles-mêmes, l'essayiste apporte un argument percutant : les femmes seraient plus conscientes que les hommes de l'artifice de l'art. S'instituant créateur, l'homme intervient et s'approprie une fonction qu'il ne possède pas, « C'est-à-dire : l'homme devient créateur parce qu'il ment, parce qu'il (se) raconte des histoires ${ }^{25}$. Sa création naît de son propre mensonge.

Contrairement au créateur masculin, la femme artiste, nous dit Huston, « n'aura pas le droit, l'autorisation, l'autorité de rejeter les hommes réels pour épouser son art, pour la bonne raison qu'elle peut produire de la vraie vie. [...] Elle fait du vrai vivant. Comment parvenir dès lors à se leurrer, au point de croire que le faux est vrai, que l'inanimé est animé, que l'esprit produit le corps ? $»^{26}$. Les hommes et les philosophes pour leur part ont longtemps refusé que la matière engendre l'intelligence, l'esprit. Et les femmes, malgré elles, se sont toujours trouvées du côté de la matière. Nancy Huston précise :

Les femmes, même lorsqu'elles désirent ardemment devenir des auteurs, sont moins convaincues de leur droit et de leur capacité à le faire. Pour la bonne raison que, dans toutes les histoires qui racontent la création, elles se trouvent non pas du côté de l'auctor (auteur, autorité), mais du côté de la mater (mère/matière).

[...]

Eh oui ! les femmes tremblent.

Peut-être parce qu'elles n'ont pas de Muses ? ou parce que, ayant si longtemps occupé sa place, elles la savent illusoire et ne parviennent pas à avoir en elles une « foi inébranlable ? $»^{27}$.

\footnotetext{
24. Ibid., p. 26.

25. Ibid., p. 23.

26. Ibid.. p. 29.

27. Ibid., p. 26 et 3 I.
} 
Mais de cette conscience de l'artifice de l'art, l'écrivaine puise justement sa force et sa puissance créatrice. Réappropriation du monde pour élaborer la fiction, mais aussi pour se rapprocher de la vie, de la vie sans mensonges, sans illusions, non plus fantasmes, mais infinies métamorphoses. Monique Larue le souligne dans son roman La Cohorte fictive. C'est une histoire partie à l'envers, la dernière-née donnant vie à la mère : « Ca ne fait rien. On fait ce qu'on veut. On la retournera comme une crêpe au moment voulu, l'histoire, qu'elle se disait, confiante $»^{28}$, consciente de l'artifice... Cette narratrice/écrivaine ambitionne de faire sortir du défilé les femmes sans nom, les «figurantes sans révolution de l'histoire ${ }^{29}$. Il s'agit d'un matrimoine difficile à écrire. Le désordre de la vie entre en contradiction avec l'ordre planifié de la fiction. Le besoin de s'affirmer par l'écriture rencontre la peur d'exister en tant que femmes, l'éclatement de celles-ci, le «je » devenant pluriel, englobant toutes les femmes. Le goût d'écrire spontanément sans la distanciation d'une narratrice/garde-fou. Écrire à même son désir.

Dans son Journal de création, Nancy Huston émet l'hypothèse que la transgression des valeurs maternelles et la révocation de la notion de normalité homme/esprit, femme/corps, permettra aux femmes de sortir des stéréotypes et du complexe d'Électre qui les poussent à valoriser l'esprit du père et à dénigrer le matériel/maternel. Le rapport des créatrices à leur mère apparaît en effet problématique, les mères entraînant les filles du côté du corps, de la procréation et les empêchant de s'affirmer comme créatrices. C'est le père qui est symbole de l'esprit; il est celui qui permet l'accès à la création. Les créatrices doivent donc se couper de leur corps puisqu'elles ne parviennent ni à transgresser ni à transcender les valeurs maternelles, et s'affirmer, du côté du père, du côté de l'« intellect », comme véritable « créateur ». Le cheminement qui les conduit sur la voie de la création les oblige à affimer comme Athéna : « Je suis née de Jupiter et je n'ai pas de mère », à refuser le maternel pour accéder à l'esprit. Mais la scission entre corps et esprit peut provoquer la maladie ou la folie, comme Francine Lemay en donne l'illustration dans son roman La Falaise (L'Hexagone, I985). La narratrice de la Cohorte fictive la prédisait déjà cette difficulté de s'affirmer dans son corps et dans son esprit, tous deux enfin réconciliés : «Elle ne sera pas facile à dire, la sensation d'exister, la joyeuse certitude d'être là ${ }^{30}$. Le plaisir/désir de l'écriture de Claire Dé et d'Anne Dandurand dans La Louve-garou (Éd. de la Pleine Lune, I98I) l'expérimente, rejoignant en cela l'écrire/vivre de Yolande Villemaire dans La Vic en prose (Les Herbes rouges, I980), ce roman du début de la décennie 80 dont Lise Potvin dit dans Voix et images : « Livre de femmels) qui ne théorise ni ne prêche mais qui met en

28. La Cohorte fictive, op. cit., p. 30.

29. Ibid., p. 43.

30. Ibid., p. 31. 
jeu une façon d'être qui est une façon d'écrire > $^{3 \mathrm{I}}$. Cet amour des mots/vie ne conduit-il pas aux mots imagés du Bruit des choses vivantes d'Élise Turcotte (Leméac, ig9ı)?

Traditionnellement, le pouvoir pro-créateur de la femme est minimisé, son rôle se réduisant à produire la « vraie » vie, animale et mortelle, alors que l'homme recrée la vie par la force de l'esprit au travers de sa création artistique et littéraire. On a trop longtemps réussi à convaincre les femmes de leur incapacité à nourrir des aspirations élevées, l'enfantement immatériel, incorporel, étant considéré comme une manifestation supérieure, plus vraie que nature. La création du monde (en sept jours) n'est-elle pas un acte de l'esprit ? Est-ce à dire que la vie est moins importante que l'art ? que les fictions de l'Histoire? Le concept de maternité littéraire s'explique par l'obligation de prouver quelque chose : l'enfant-chair ou le livre-peau : «Quand j'aurai prouvé ceci, je pourrai me permettre cela $>^{32}$, dira Huston.

Les écrivaines des années quatre-vingt, nous l'avons vu dans ce bref survol, semblent en quête d'un nouveau rapport à la vie, conciliant l'activité de l'esprit et la matérialité de l'être. Par une appréhension autre, en ayant le désir constant d'élargir leur démarche d'écriture, les femmes explorent de nouvelles voies/voix. De la dichotomie création/procréation, point de départ de cette réflexion qui ne fait que reprendre le questionnement éthique/esthétique, les écrivaines tentent de redéfinir l'acte créateur, en privilégiant une écriture polysémique, en créant des représentations multiples, plurielles, des images contradictoires, ambivalentes, comme la vie elle-même, de tout ces petits riens qui font précisément... l'existence.

\section{Le complexe d'lcare par Chantal Théry}

Les écrivaines veulent tout, l'esprit et le corps à la fois, la vie d'abord, la fiction ensuite, un discours théorique et incarné, qui prend en compte le réel ${ }^{33}$ !

Dans son article, « Il n'y a pas de cogito-femme », publié dans le livre collectif LÉmergence d'une culture au féminin ${ }^{34}$, Françoise Collin perçoit avec raison et urgence que les rapports hommes-femmes ne peuvent échapper à un rapport réinventé à la mère :

31. Voix et Images, printemps ig86, p. 406.

32. «Car le cela, loin de se contenter de prélever du temps au ceci, abolit jusqu'au je qui pouvait, telle une balance, tenir un enfant dans une main et un livre dans l'autre », Nancy Huston, op. cit., P. I4I-142.

33. A lire aussi : Chantal Chawaf, le Corps et le verbe. La langue en sens inverse, les Essais, Presses de la Renaissance, 1992.

34. Françoise Collin, dans L'Emergence d'une culture au féminin, sous la direction de Marisa Zavalloni, Montréal, Editions Saint-Martin, 1987, p. 107-ı16. 
La culture au féminin devrait être au moins une culture «douce », comme on dit qu'il y a des énergies douces. Alors que la culture masculine, à travers les rites directs ou déguisés de l'initiation, contraint les hommes à renoncer à leurs origines, à la langue maternelle dans laquelle ils ont d'abord été formés et commence donc par une mise à mort qu'elle ne cessera de répéter, la culture au féminin évite ce traumatisme : elle développe et consolide les origines en les dépassant et maintient un lien constant de la maturité avec l'enfance. Elle ne part pas d'un meurtre, ni d'un suicide, ni d'une oblitération honteuse de la mémoire. [...]

Car pour ce qui est de la socialisation et de la culture, les hommes ont au contraire à se trancher d'eux-mêmes avec violence en renonçant au polymorphisme de leurs premières années : l'accès au social est institué comme un saut qualitatif irréversible par rapport au privé et à l'enfance qui repose sur le matricide. La socialisation des femmes et leur culture ne se pensent pas dans cette coupure. C'est pourquoi, sans doute, elle a quelques affinités avec ce qu'on nomme aujourd'hui l'écologie : une écologie de la nature qui est aussi une écologie de l'esprit.

Aujourd'hui, ce qui ressort de nos recherches, c'est que les đEdipe modernes en Crise de masculinité semblent moins du côté de Jocaste que du côté de Laïos, enclins à regretter le parricide, à ne pouvoir retrouver la mère qu'en réinventant les rapports au père, à ne pouvoir réinventer la mère, qu'en retrouvant le père.

Les hommes, hybrides malheureux, en quête de métamorphoses, d'identités plurielles, itinérants sans frontières, hermaphrodites-autonomes errant avec leurs carapaces sur le dos (c'est, par analogie, la roulotte dans Salut Galameau, le manteau de Sol qui lui tient lieu de maison, de valise, de pays, d'affection, la Volkswagen Blues ou la maison du Vieux chagrin de Jacques Poulin, etc.). Ces personnages masculins, nostalgiques d'un monde utérin qu'ils tentent de réinvestir à leur façon, souffrent du complexe du scaphandrier ou du complexe de Jonas : souvenez-vous... Gepetto, nouveau père, ne se réconciliera avec son fils Pinocchio que dans le ventre de la mer/de l'a-mère baleine, qu'en témoignant enfin à son fils toute sa tendresse, ayant apprivoisé le mal de mère, nouvel adepte, à sa façon, du « care ».

La quête des pères, des nouveaux pères, le père maternant/« nurturant » son fils ou sa fille, ou le fils maternant son père, se manifeste de plus en plus dans les œuvres récentes. Il s'agit bien de réinventer la douceur et la tendresse au masculin. Des mots sont aussi à inventer pour ne plus associer le doux et tendre maternage à la mère : comment traduire en français « nursing » et « nurturing » en les dissociant du rôle de sexe, des femmes-mères auxquelles ils se rattachent socialement et non ontologiquement, comme le souligne bien Francine Saillant?

En cette fin de $\mathrm{XX}^{\mathrm{e}}$ siècle, le masculin en crise se rejoue la tragédie d'Icare: faute d'aimer son corps et de se satisfaire de l'histoire dite sans qualité de la Vie matérielle, quotidienne, Icare s'invente des ailes à sa démesure, artificiellement collées, prend son envol vers Dieu-le-Père-Soleil, les Cieux, le pur Esprit, précipitant finalement dans sa chute, moins son suprême orgueil de mâle en mal de désincarnation que l'espoir d'une humanité enfin retrouvée, 
d'ennemi/e/s réconcilié/e/s, Corps-Esprit sans scission ni hiérarchie. A l'orée du XXI siècle, Icare ne peut plus, dans une société nouvelle, que s'écrire « He cares »!... Les « Icare-machas » de notre fin XXe siècle, battantes et souffrantes pour mieux performer, briller et s'intégrer au monde des hommes, ne pourront plus non plus, après s'être méfiées à juste titre de l'aliénation des mèrespélicans, que réinventer un « care » à la mesure des deux sexes.

« Jai une âme. Je sens que j'ai une âme! Une âme prise dans mon corps, pas une âme de catéchisme! », écrit un Robert Lalonde qui tente de se déprendre des rets, de la fêlure idéologique du catholicisme :

plus tard, mon amour, on essaiera de me faire comprendre que d'un côté de ma peau, il y aura Dieu, et que de l'autre, il y aura moi, que nous ne pourrons jamais nous rencontrer, lui et moi. Il ne rencontrera donc jamais mon cœur, mes poumons, ma cervelle, Dieu ? Ni les arbres sous leur écorce, ni le papillon dans son cocon ? Je saurai qu'on me ment. Je suis un morceau de ce Dieu, une étincelle échappée d'un soleil qui n'en finit pas d'exploser et de libérer des étoiles qui restent incandescentes longtemps. Leur Dieu ne sera pas le mien. Dans mes limbes, mon Dieu est mâle et femelle, sensible, palpable. C'est le désir sans fin et aussi l'assouvissement qui viendra, mystérieux et sûr à la fois. La paix asphyxiante des églises, le paradis toujours promis, espéré, jamais souhaitable, la séparation du monde entre le bien et le mal, entre les gestes sages et les gestes fous, entre l'aubier et l'écorce : pas pour moi. Je n'appartiendrai pas au troupeau des êtres sans grâce qui cherchent l'oubli. Tout de suite, là, maintenant, il y a la passion bien suffisante, l'appétit, l'attente et le déliement, la vie. J'ai déjà l'immense conviction que ce sera bien assez que de n'être plus dans la mort. Assez pour triompher même dans la douleur. Assez pour aimer, même dans le doute. Assez pour ne pas vouloir y retourner de sitôt, dans la mort. Ni philosophie, ni morale. Une certitude de limbes et bientôt de chair, une croyance rouge et battante : je m'en viens, hors de la mort, propulsé, pour ne rien savoir d'autre que cette suite des limbes, cette insubordination à la loi des curés et des obtus, cette limoidité sans indulgence ni pardon, cette échappée hors de la nuit, cet éclat parfois déchirant, ce dieu, le grand jour. (p. 166-167)

L'un de ses personnages masculins se laissera aller, doucement balancé dans une barque, à respirer toutes les odeurs et à « sentir la fleur veloutée d'un songe féminin s'ouvrir dans son ventre. » La Belle au bois dormant a changé d'histoire et de sexe... Aujourd'hui, dans le Beau au bois dormant, la fleur veloutée d'un tendre songe doit, réellement, s'ouvrir. 